\title{
RESISTÊNCIA À IVERMECTINA EM POPULAÇÕES DE MUSCA DOMESTICA (DIPTERA: MUSCIDAE) PROVENIENTES DE GRANJAS DE GALINHAS POEDEIRAS
}

\author{
C.M.G. Ambrós; A.P. Prado
}

UNICAMP, Instituto de Biologia, Departamento de Parasitologia, Laboratório de Entomologia, Rua Monteiro Lobato, 255, CEP 13083-862, Campinas, SP, Brasil. E-mail: cginarte@hotmail.com

\section{RESUMO}

\begin{abstract}
Bioensaios concentração-resposta foram realizados com o objetivo de estudar o efeito larvicida e detectar possível resistência à ivermectina em dez populações de $M$. domestica provenientes de granjas de galinhas poedeiras. Para tal finalidade, o meio de cultura para larvas foi tratado com diferentes concentrações de ivermectina $(0,015 ; 0,03 ; 0,06 ; 0,125 ; 0,25$ e 0,5 ppm). Atividade larvicida da ivermectina e anomalias nas pupas foram detectadas. A população mais resistente à ivermectina foi Monte Mor, $\mathrm{SP}\left(\mathrm{CL}_{50}=0,262 \mathrm{ppm}\right.$ e $\left.\mathrm{FR}=13,1\right)$. As populações de Campinas, $\mathrm{SP}\left(\mathrm{CL}_{50}=0,141 \mathrm{ppm}\right.$ e FR = 7,1), Promissão, SP $\left(\mathrm{CL}_{50}=0,110\right.$ ppm e FR = 5,5), Santa Cruz da Conceição, $\mathrm{SP}\left(\mathrm{CL}_{50}=0,102\right.$ ppm e FR = 5,1), Holambra, SP $\left(\mathrm{CL}_{50}=0,071\right.$ ppm e $\left.\mathrm{FR}=3,6\right)$ e Hortolândia, $\mathrm{SP}\left(\mathrm{CL}_{50}=0,068\right.$ ppm e $\mathrm{FR}=3,4)$ foram mais resistentes que as de Sumaré, $\mathrm{SP}\left(\mathrm{CL}_{50}=0,052 \mathrm{ppm}\right.$ e $\left.\mathrm{FR}=2,6\right)$, Sarapuí, $\mathrm{SP}\left(\mathrm{CL}_{50}\right.$ $=0,050 \mathrm{ppm}$ e $\mathrm{FR}=2,5)$ e Itanhandu, $\mathrm{MG}\left(\mathrm{CL}_{50}=0,047 \mathrm{ppm}\right.$ e $\left.\mathrm{FR}=2,4\right)$. A população mais susceptível à ivermectina foi Grande Porto Alegre, $\mathrm{RS}\left(\mathrm{CL}_{50}=0,020 \mathrm{ppm}\right.$ e $\left.\mathrm{FR}=1,0\right)$. Os resultados indicam que a ivermectina pode ser eficaz contra larvas deM. domestica. Entretanto, resistência foi suspeitada em algumas das populações, sendo importante a realização de novos estudos nesses locais.
\end{abstract}

PALAVRAS-CHAVE: Inseticida, bioensaio, suscetibilidade, controle de insetos.

\section{ABSTRACT}

RESISTANCE TO IVERMECTIN IN POPULATIONS OF MUSCA DOMESTICA (DIPTERA: MUSCIDAE) FROM POULTRY FARMS. Larvicidal effect and resistance to ivermectin in 10 populations of Musca domestica from poultry farms was evaluated by concentration-response bioassays. For this purpose, the larval growth medium was treated with different concentrations of ivermectin $(0.015,0.03,0.06,0.125,0.25$ and $0.5 \mathrm{ppm})$. As a result, larvicidal activity of ivermectin was observed and some anomalies were detected in pupae. The population from Monte Mor, SP, was the most resistant to ivermectin $\left(\mathrm{LC}_{50}=0.262 \mathrm{ppm}\right.$ and $\left.\mathrm{RF}=13.1\right)$. Fly populations from Campinas, $\mathrm{SP}\left(\mathrm{LC}_{50}=0.141 \mathrm{ppm}\right.$ and $\left.\mathrm{RF}=7.1\right)$, Promissão, $\mathrm{SP}\left(\mathrm{LC}_{50}=0.110 \mathrm{ppm}\right.$ and $\left.\mathrm{RF}=5.5\right)$, Santa Cruz da Conceição, SP $\left(\mathrm{LC}_{50}=0.102 \mathrm{ppm}\right.$ and $\left.\mathrm{RF}=5.1\right)$, Holambra, SP $\left(\mathrm{LC}_{50}=0.071 \mathrm{ppm}\right.$ and $\mathrm{RF}$ $=3.6)$ and Hortolândia, $\mathrm{SP}\left(\mathrm{LC}_{50}=0.068 \mathrm{ppm}\right.$ and $\left.\mathrm{RF}=3.4\right)$ were more resistant than the ones from Sumaré, $\mathrm{SP}\left(\mathrm{LC}_{50}=0.052 \mathrm{ppm}\right.$ and $\left.\mathrm{RF}=2.6\right)$, Sarapuí, $\mathrm{SP}\left(\mathrm{LC}_{50}=0.050 \mathrm{ppm}\right.$ and $\left.\mathrm{RF}=2.5\right)$, and Itanhandu, $\mathrm{MG}\left(\mathrm{LC}_{50}=0.047 \mathrm{ppm}\right.$ and $\left.\mathrm{RF}=2.4\right)$. The population most susceptible to ivermectin was that from Grande Porto Alegre, $\mathrm{RS}\left(\mathrm{LC}_{50}=0.020 \mathrm{ppm}\right.$ and $\left.\mathrm{RF}=1.0\right)$. The results indicate that ivermectin can be effective against larvae of $M$. domestica. However, since resistance was suspected in some of the populations studied, it is important to carry out new studies in these places.

KEY WORDS: Insecticide, bioassay, susceptibility, insect control.

\section{INTRODUÇÃO}

Musca domestica L.éuma espécie de grandeimportância econômica e em saúde pública, por ser vetor mecânico de patógenos e uma importante praga nas granjas de galinhas e criações de gado, afetando a produtividade GreenberG, 1971; Axtell; Arends, 1990). Vários métodos de controle têm sido empregados para controlar esta praga, sendo o uso de inseticidas químicos o mais utilizado, o que tem levado ao desenvolvimento da resistência Metcalf, 1980; BLOOMCAMP et al., 1987). Esses fatores têm conduzido à busca de novas substâncias, dentre as quais se encontram as avermectinas.

As avermectinas são um subgrupo das lactonas macrocíclicas muito efetivas contra nematoides e artrópodos, incluindo insetos, ácaros e carrapatos (PutTeretal.,1981). Aivermectina éum dos principais representantes deste grupo e seu modo de ação envolve o ácido gama-aminobutírico (GABA), um 
neurotransmissor que emite sinais entre células nervosas ou entre estas e os músculos. Em artrópodes, o estímulo mediado pelo GABA interrompe o impulso nervoso entre a terminação nervosa e o músculo, produzindo paralisia e morte (STRONG; BROWN, 1987).

Vários estudos têm demonstrado o efeito da ivermectina em larvas de dípteros. KRÜGER; SCHOLTZ (1995) observaram inibição na emergência e uma redução na fertilidade deMusca nevilli Kleynhans (Diptera: Muscidae) em fezes de bovinos tratados com ivermectina. BYFORD etal. (1999) obtiveram resistência a ivermectina em Haematobia irritans (Linnaeus, 1758) (Diptera:Muscidae) apóscerca de 30 gerações mantidas sob pressão de seleção com esse inseticida. Bioensaios realizados com larvas de $M$. domestica, em fezes de bovinos e suínos tratados com ivermectina, doramectina e moxidectina injetáveis, mostraram reduzida emergência sendo o efeito larvicida dessas drogas observado durante 3 a 4 semanas nas fezes bovinas (FARKAS etal.,2003). Ivermectina numa concentração de 1,5 ppb causou paralisia em larvas de Culex quinquefasciatus (Diptera: Culicidae), com mortalidade de 73,38\% (Alves etal., 2004). Lima etal.(2009) observaram ausência de $H$. irritans durante 28 dias, em amostras fecais de bovinos tratados com ivermectina.

As avermectinas são usadas amplamente no Brasil no controle de ecto e endoparasitas no gado, sendo gastos cerca de 300 milhões de reais em 2006 (SINDICAto Nacional da Indústria de Produção para Saúde ANIMAL, 2007). A presente pesquisa objetivou avaliar a atividade larvicida da ivermectina em $M$. domestica e determinar possível resistência em dez populações provenientes de granjas de galinhas poedeiras em São Paulo, Minas Gerais e Rio Grande doSul, por meio de bioensaios concentração-resposta.

\section{MATERIAL E MÉTODOS}

Larvas de $M$. domestica foram amostradas em granjas degalinhas poedeiras de diferentes localidades dosestados de São Paulo, Minas Gerais e Rio Grande do Sul. As coletas de campo foram realizadas no período de janeiro de 2000 a dezembro de 2002, acompanhadas da caracterização dos locais em relação ao histórico de inseticidas utilizados(Tabela1). Aslarvasforam coletadas comuma pá diretamente das fezes de galinhas e acondicionadas em potes plásticos $(20 \times 15 \times 5 \mathrm{~cm})$, sendo levadas posteriormenteao Laboratório de Entomologia, Departamento de Parasitologia (IB) da Universidade Estadual de Campinas para sua manutenção e criação. Os adultos emergidos eram colocadosem gaiolas plásticas $(25 \mathrm{~cm} \times 35 \mathrm{~cm}$ x $30 \mathrm{~cm}$ ), com aberturas recobertas com tela de "nylon" para permitir a aeração e mantidos em uma câmara de germinação a uma temperatura $\left(27 \pm 1^{\circ} \mathrm{C}\right)$, umidade relativa $(70 \%)$ e fotoperíodo $(12 / 12)$ controlados. A ali- mentaçãodosadultosconsistiudeumamisturadepartes iguais deleiteem póintegral, açúcarelevedura decerveja. Água e alimento eram oferecidos e mantidos em abundância dentro das gaiolas.

As gerações de $M$. domestica obtidas no laboratório, a partirdageração $\mathrm{F}_{2}$, foram utilizadas nos bioensaioscom formulação comercial de ivermectina (Ivomec $\left.{ }^{\circledR} 1 \% \mathrm{p} / \mathrm{v}\right)$.

Para a obtenção das larvas de $M$. domestica, utilizadas na realização dos bioensaios, foram retiradas oviposições feitas em organza preta embebida em uma solução composta por $60 \mathrm{~mL}$ de leite integral, $2 \mathrm{~g}$ de levedura de cerveja e 1,6 g de bicarbonato de amônia. Os ovos foram transferidos com ajuda de um pincel para recipientes com papel de filtro umedecido com água até a eclosão das larvas.

Para a realização dos bioensaios, o meio cultura para larvas de $M$. domestica foi tratado com diferentes concentrações de ivermectina. $\mathrm{O}$ meio utilizado foi constituído por: 12,56 partes de farelo de trigo, 1 parte delevedo decerveja, 1,2 partes deleite em pó,36 partes de água declorada e 0,26 partes de nipagin (antifúngico). As concentrações de ivermectina utilizadas foram: 0,$015 ; 0,03 ; 0,06 ; 0,125 ; 0,25$ e 0,5 ppm.

Os bioensaios foram efetuados em copos plásticos ( $200 \mathrm{~mL}$ ) contendo $50 \mathrm{~g}$ do meio de cultura misturado com o inseticida nas diferentes concentrações, onde foram colocadas 30 larvas de primeiro estádio por copo. Foram feitas quatro réplicas para cada tratamento e um grupo controle para cada réplica, no qual no foi adicionado inseticida. Os copos plásticos foram tampados, tendo a tampa uma abertura de $1,5 \mathrm{~cm}$ protegida por organza, para permitir a aeração e evitar a saída dos insetos.

Os bioensaios foram mantidos em câmara de germinação com temperatura $\left(26 \pm 1^{\circ} \mathrm{C}\right)$, umidade relativa (>60\%) e fotoperíodo $(12 / 12)$ controlados. Após nove dias, as pupas formadas eram extraídas do meio e mantidas em câmara até a emergência dos adultos. Foi realizada a contagem dos sobreviventes que conseguiam emergir por completo do pupário.

Os dados foram analisados estatisticamente utilizando-se o PROC GLM do programa estatístico SAS (Statistical Analysis System) (SAs Institute, 1986) para a realização de ANOVA. As comparações entre as médias foram feitas pelo teste de comparações múltiplas de Duncan. Foi realizada análise de probit (FINNEY, 1971) para o cálculo das concentrações letais para $50 \%$ e $95 \%$ dos insetos tratados $\left(\mathrm{CL}_{50} \mathrm{eCL}_{95}\right)$ comoprograma Polo PC (Leora SofWAre, 1987). Foi considerado o nível de significância $\alpha=0,05$. O Fator de Resistência foi calculado dividindo-se a $\mathrm{CL}_{50}$ de cada população testada pela $\mathrm{CL}_{50}$ da população com menor valor de $\mathrm{CL}_{50}$ dentre as dez populações testadas. Foram consideradas resistentes as populações com Fator de Resistência maior que 1, de acordo com o critério adotado por IsEKI; Georghiou (1986) e KeIDING (1999). 
Tabela 1 - Caracterização dos locais de coleta de Musca domestica e histórico de inseticidas utilizados nas granjas.

\begin{tabular}{|c|c|c|c|}
\hline Localidade & Coordenadas & Estado & Inseticidas utilizados \\
\hline Grande Porto Alegre & $\begin{array}{l}30^{\circ} 01^{\prime} 59^{\prime \prime} \mathrm{S} \\
51^{\circ} 13^{\prime} 48^{\prime \prime} \mathrm{W}\end{array}$ & Rio Grande do Sul & Cyromax (ciromazina) \\
\hline Itanhandu & $\begin{array}{l}22^{\circ} 17^{\prime} 45^{\prime \prime} \mathrm{S} \\
44^{\circ} 56^{\prime} 05^{\prime \prime} \mathrm{W}\end{array}$ & Minas Gerais & Cyromax (ciromazina) \\
\hline Sarapuí & $\begin{array}{l}23^{\circ} 38^{\prime} 26^{\prime \prime} \mathrm{S} \\
47^{\circ} 49^{\prime} 29^{\prime \prime} \mathrm{W}\end{array}$ & São Paulo & $\begin{array}{l}\text { Nuvan (diclorvós) } \\
\text { Isca Vetor (azametifós) } \\
\text { Larvadex (ciromazina) }\end{array}$ \\
\hline Sumaré & $\begin{array}{l}22^{\circ} 49^{\prime} 13^{\prime \prime} \mathrm{S} \\
47^{\circ} 16^{\prime} 08^{\prime \prime} \mathrm{W}\end{array}$ & São Paulo & $\begin{array}{l}\text { Isca Vetor (azametifós) } \\
\text { Larvadex (ciromazina) } \\
\text { Cyromax (ciromazina) }\end{array}$ \\
\hline Hortolândia & $\begin{array}{l}22^{\circ} 51^{\prime} 22^{\prime \prime} \mathrm{S} \\
47^{\circ} 13^{\prime} 05^{\prime \prime} \mathrm{W}\end{array}$ & São Paulo & $\begin{array}{l}\text { Sanemosca larvicida (diclorvós }+0,0 \text { dimetil S } \\
\text { (nmetilcarbamoyl metilfosforiditioate) }\end{array}$ \\
\hline Holambra & $\begin{array}{l}22^{\circ} 37^{\prime} 55^{\prime \prime} \mathrm{S} \\
47^{\circ} 03^{\prime} 36^{\prime \prime} \mathrm{W}\end{array}$ & São Paulo & $\begin{array}{l}\text { Azodrin (monocotrofós) } \\
\text { Isca Vetor (azametifós) }\end{array}$ \\
\hline Santa Cruz da Conceição & $\begin{array}{l}22^{\circ} 08^{\prime} 25^{\prime \prime} \mathrm{S} \\
47^{\circ} 27^{\prime} 07^{\prime \prime} \mathrm{W}\end{array}$ & São Paulo & $\begin{array}{l}\text { Sanemosca larvicida (diclorvós+0,0 Dimetil S } \\
\text { (nmetilcarbamoyl metilfosforiditioate) } \\
\text { Isca Vetor (azametifós) }\end{array}$ \\
\hline Promissão & $\begin{array}{l}21^{\circ} 32^{\prime} 12^{\prime \prime} \mathrm{S} \\
49^{\circ} 51^{\prime} 29^{\prime \prime} \mathrm{W}\end{array}$ & São Paulo & $\begin{array}{l}\text { Nuvan (diclorvós) } \\
\text { Isca Vetor (azametifós) } \\
\text { Larvadex (ciromazina) } \\
\text { Cyromax (ciromazina) }\end{array}$ \\
\hline Campinas & $\begin{array}{l}22^{\circ} 53^{\prime} 20^{\prime \prime} \mathrm{S} \\
47^{\circ} 04^{\prime} 40^{\prime \prime} \mathrm{W}\end{array}$ & São Paulo & $\begin{array}{l}\text { Sanemosca larvicida (diclorvós }+0,0 \text { dimetil S } \\
\text { (nmetilcarbamoyl metilfosforiditioate) }\end{array}$ \\
\hline Monte Mor & $\begin{array}{l}22^{\circ} 56^{\prime} 47^{\prime \prime} \mathrm{S} \\
47^{\circ} 18^{\prime} 58^{\prime \prime} \mathrm{W}\end{array}$ & São Paulo & DDVP (diclorvós) \\
\hline
\end{tabular}

\section{RESULTADOS E DISCUSSÃO}

Os dados de concentração-resposta para as dez populações estudadas são mostrados na Tabela 2. A população mais suscetível foi a proveniente de GrandePorto Alegre, $\mathrm{RS}$, com $\mathrm{CL}_{50}$ de 0,020 ppm, tendo sido utilizada como referência para o cálculo dos Fatores de Resistência (FR). As $\mathrm{CL}_{50}$ das dez populações de $M$. domestica estudadas estiveram na faixa de 0,02 - 0,26 ppm, sendo semelhantes às $\mathrm{CL}_{50}$ obtidas por ALBRECHT; SHERMAN (1987) ao realizar aplicação tópica de avermectina $B_{1}$ em fêmeas de três espécies de moscas das frutas: Dacus dorsalis (Diptera: Tephritidae) $\left(\mathrm{CL}_{50}\right.$ $=0,021 \mathrm{ppm}$ ), Dacus cucurbitae (Diptera: Tephritidae) $\left(\mathrm{CL}_{50}=0,042 \mathrm{ppm}\right)$ e Ceratitis capitata (Diptera: Tephritidae) $\left(\mathrm{CL}_{50}=0,29 \mathrm{ppm}\right)$.

Amaior $\mathrm{L}_{50}$ foia da população deMonteMor,SP(FR =13,1), sendo, por tanto, a mais resistenteà ivermectina, seguida de Campinas, SP, Promissão, SP e Santa Cruz da Conceição, SP (com FR entre 5 e 10). À continuação pode-seobservaroutrogrupo (populações deHolambra, SP e Hortolândia, SP) com menores valores de concen- trações letais e FR entre 3 e 5, entretanto, Sumaré, SP, Sarapuí,SPeItanhandu, MG foram as mais suscetíveis, com fatores de resistência menores de 3 .

Em nenhuma das dez populações estudadas no presente trabalho tinha sido utilizada ivermectina anteriormente; nas quatro populações mais resistentes (Monte Mor, SP, Campinas, SP, Promissão, SP e Santa Cruz da Conceição, SP) o controle de moscas era realizado mediante a utilização de inseticidas organofosforados, carbamatos e o regulador do crescimento de insetos ciromazina. Roush; WRIGHT (1986) não encontraram resistência à abamectina, outra avermectina, em seis populações de $M$. domestica resistentes ao DDT, dieldrin, organofosforados e piretroides, entretanto, Scott (1989) e Geden et al. (1992) encontraram resistência à abamectina em populações de campo resistentes à permetrina. RuGG et al. (1998) relataram resistência cruzada com moxidectina (milbemicina), mas não com diazinon e cipermetrina, numa população de Lucilia cuprina (Diptera: Calliphoridae) submetida sob pressão de seleção com ivermectina. 
Tabela 2 - Suscetibilidade à ivermectina em larvas de diferentes populações de Musca domestica provenientes de granjas de galinhas poedeiras, coletadas de janeiro/2000 a dezembro/2002.

\begin{tabular}{|c|c|c|c|c|}
\hline Populações & $\begin{array}{c}\mathrm{CL}_{50} \\
\text { (IC a } 95 \%)\end{array}$ & $\begin{array}{c}\mathrm{CL}_{95} \\
(\mathrm{IC} \text { a } 95 \%)\end{array}$ & $\mathrm{b} \pm$ desvio padrão & FR \\
\hline Grande Porto Alegre, RS* & $\begin{array}{l}0,020 \\
(0,013-0,027)\end{array}$ & $\begin{array}{l}0,421 \\
(0,282-0,746)\end{array}$ & $1,234 \pm 0,134$ & 1,0 \\
\hline Itanhandu, MG & $\begin{array}{l}0,047 \\
(0,035-0,058)\end{array}$ & $\begin{array}{l}0,265 \\
(0,203-0,379)\end{array}$ & $2,183 \pm 0,228$ & 2,4 \\
\hline Sarapuí, SP & $\begin{array}{l}0,050 \\
(0,042-0,059)\end{array}$ & $\begin{array}{l}0,166 \\
(0,136-0,218)\end{array}$ & $3,157 \pm 0,326$ & 2,5 \\
\hline Sumaré, SP & $\begin{array}{l}0,052 \\
(0,033-0,070)\end{array}$ & $\begin{array}{l}0,348 \\
(0,252-0,572)\end{array}$ & $1,992 \pm 0,226$ & 2,6 \\
\hline Hortolândia, SP & $\begin{array}{l}0,068 \\
(0,050-0,087)\end{array}$ & $\begin{array}{l}0,600 \\
(0,424-0,982)\end{array}$ & $1,739 \pm 0,189$ & 3,4 \\
\hline Holambra, SP & $\begin{array}{l}0,071 \\
(0,047-0,091)\end{array}$ & $\begin{array}{l}0,279 \\
(0,214-0,444)\end{array}$ & $2,765 \pm 0,411$ & 3,6 \\
\hline Santa Cruz da Conceição, SP & $\begin{array}{l}0,102 \\
(0,079-0,124)\end{array}$ & $\begin{array}{l}0,475 \\
(0,374-0,675)\end{array}$ & $2,462 \pm 0,295$ & 5,1 \\
\hline Promissão, SP & $\begin{array}{l}0,110 \\
(0,087-0,134)\end{array}$ & $\begin{array}{l}0,816 \\
(0,585-1,321)\end{array}$ & $1,892 \pm 0,201$ & 5,5 \\
\hline Campinas, SP & $\begin{array}{l}0,141 \\
(0,116-0,163)\end{array}$ & $\begin{array}{l}0,348 \\
(0,291-0,467)\end{array}$ & $4,199 \pm 0,636$ & 7,1 \\
\hline Monte Mor, SP & $\begin{array}{l}0,262 \\
(0,201-0,337)\end{array}$ & $\begin{array}{l}1,847 \\
(1,111-4,769)\end{array}$ & $1,940 \pm 0,320$ & 13,1 \\
\hline
\end{tabular}

$\mathrm{CL}_{50}=$ concentração letal em ppm para $50 \%$ dos insetos tratados.

$\mathrm{CL}_{95}=$ concentração letal em ppm para $95 \%$ dos insetos tratados.

IC = Intervalos de Confiança.

$\mathrm{b}=$ coeficiente angular da reta.

$\mathrm{FR}=$ Fator de Resistência $\left(\mathrm{CL}_{50}\right.$ da população/ $\mathrm{CL}_{50}$ da população susceptível de referência).

*população mais susceptível, usada como referência no cálculo dos fatores de resistência (FR).

As diferenças encontradas quanto à suscetibilidade das populações pesquisadas podem ser devidas a vários fatores. Segundo KeIDING (1999), o desenvolvimento da resistência a inseticidas depende de fatores genéticos, biológicos eoperacionais, além disso, variações estacionais na suscetibilidade de $M$. domestica a inseticidas têm sido registradas por Oliveira et al. (1993). Eventos de extinção e recolonização podem incrementar a diferenciação genética entre populações (CAPRIO; HOY, 1994).

Em relação à inclinação da reta, para a população mais resistente (Monte Mor, SP) dentre as dez populações estudadas, ocoeficiente angular foi baixo $(1,94)$, entretanto, a outra população que lhe segue em resistência (Campinas, SP) teve um coeficiente angular alto $(4,20)$, o que pode indicar uma maior homogeneidade da população. O coeficiente angular poderia ser um indicativo da variabilidade genética dentro da população: quanto menor seu valor, maior heterogeneidade (BROWN; PAL, 1971). IsEKI; GEORGHIOU (1986) também comentaram sobre ofato de que o baixo coeficiente angular da reta em uma população de $M$. domestica tratada com ciromazina poderia indicar uma maior heterogeneidade da população. A população mais suscetível (Rio Grande do Sul, RS) foi a mais heterogênea dentre as amostradas por apresentar menor coeficiente angular $(1,23)$, pelo que a maior suscetibilidade desta população à ivermectina pode ser decorrente da ocorrência de mecanismos de resistência menos eficientes ou da baixa frequência de indivíduos resistentes a este princípio ativo e não da ausência destes na população.

Nas dez populações pesquisadas houve um aumento da mortalidade com a concentração do inseticida, observando-se diferenças significativas em relação à mortalidade nas diferentes concentrações testadas [Grande Porto Alegre, RS ( $F=175,7 ; p<0,0001)$; Santa Cruz da Conceição, SP ( F = 116,8; $\mathrm{p}<0,0001)$; Sumaré, $\mathrm{SP}(\mathrm{F}=124,0 ; \mathrm{p}<0,0001)$; Monte Mor, $\mathrm{SP}$ (F $=50,8 ; \mathrm{p}<0,0001)$; Itanhandu, MG $(\mathrm{F}=132,1 ; \mathrm{p}<$ 0,0001); Hortolândia, SP (F = 95,6; p < 0,0001); Campinas, $\mathrm{SP}(\mathrm{F}=110,1 ; \mathrm{p}<0,0001)$; Promissão, $\mathrm{SP}(\mathrm{F}=$ 165,4; $\mathrm{p}<0,0001)$; Holambra, $\mathrm{SP}(\mathrm{F}=333,3 ; \mathrm{p}<0,0001)$ e Sarapuí, SP $(\mathrm{F}=277,2 ; \mathrm{p}<0,0001)]$. Pesquisas realizadas por MASKARI et al.(2008) indicaram mortalidade larval de Plebotomus papatasi (Diptera: 
Psychodidae) de 100\% nas concentrações mais altas desse inseticida. Resultados similares foram encontrados por Silva; Mendes (2002) para larvas de $H$. irritans tratadas com concentrações na faixa de 20 a 300 ppb de diflubenzuron.

Devido à ação da ivermectina foram encontradas algumas pupas deformadas e menores que as normais. Este fato foi também observado por PEDROSO-DEPaIva; Goya (1994) em Sarcopromusca pruna (Diptera: Muscidae) criada em fezes de animais tratados com ivermectina, encontrando pupas anômalas, com pouca quitinização da cutícula e que não evoluíram para adultos.

De modo geral, a ivermectina mostrou-se eficaz em baixas concentrações contra larvas de $M$. domestica, embora a suscetibilidade a este inseticida tenha variado nas diferentes populações estudadas. Deve-se salientar que resistência foi suspeitada em algumas das populações estudadas, motivo pelo qual seria importante a realização de novos estudos nos respectivos locais.

\section{AGRADECIMENTOS}

À CAPES pela bolsa de doutorado.

\section{REFERÊNCIAS}

ALBRECHT, C.P.; SHERMAN, M. Lethal and sublethal effects of avermectin $B_{1}$ on three fruit fly species (Diptera: Tephritidae). Journal of Economic Entomology, v.80, p. 344-347, 1987.

ALVES, S.N.; SERRÃO, J.E; MOCELIN, G.; DE MELO, A.L. Effect of ivermectin on the life cycle and larval fat body of Culex quinquefasciatus. Brazilian Archives of Biology and Technology, v. 47, p.16-27, 2004.

AXTELL, R.C.; ARENDS, J.J. Ecology and management of arthropod pest of poultry. Annual Review of Entomology, v.35, p.101-126, 1990.

BLOOMCAMP, C.L.; PATTERSON, R.S.; KOEHLER, P.G. Cyromazine resistance in the house fly (Diptera: Muscidae). Journal of Economic Entomology, v.80, p.352357, 1987.

BROWN, A.W.A.; PAL, R. (Ed.). Insecticide resistance in arthropods. 2.ed. Geneva: World Health Organization, 1971. 67p.

BYFORD, R.L; CRAIG, M.E.; DEROGUEN, S.M.; KIMBALL, M.D.; MORRISON, D.G.; WYATT, W.E.; FOIL, L.D. Influence of permethrin, diazinon and ivermectin treatments on insecticide resistance in the horn fly (Diptera: Muscidae). International Journal for Parasitology, v.29, p.125-135, 1999.
CAPRIO, M.A.; HOY, M.A. Metapopulation dynamics affect resistance development in the predatory mite, Metaseiulus occidentalis (Acari: Phytoselidae). Journal of Economic Entomology, v.87, p525-534, 1994.

FARKAS, R.; GYURCSO, A.; BORZSONYI, L. Fly larvicidal activity in faeces of cattle and pigs treated with endectocide products. Medical and Veterinary Entomology, v.17, n.3, p.301-306, 2003.

FINNEY, D.J. (Ed.). Probit analysis. 3.ed. Cambridge: Cambridge University Press, 1971. 255p.

GEDEN, C.J.; RUTZ, D.A.; SCOTT, J.C.; LONG, S.J. Susceptibility of house flies and five pupal parasitoids to abamectin and seven commercial insecticides. Journal of Economic Entomology, MD, v.85, p.435-440, 1992.

GREENBERG, G. Flies and disease. Ecology, Classification and biotic associatics. Princeton: Princeton Univ. Press, 1971. 865p.

ISEKI, A.; GEORGHIOU, G. Toxicity of cyromazine to strains of the housefly variously resistant to insecticides. Journal of Economic Entomology, v.79, p.11921195, 1986.

KEIDING, J. Review of the global status and recent development of insecticide resistance in field populations of the housefly, Musca domestica (Diptera: Muscidae). Bulletin of Entomological Research, v.89, p.S7S67, 1999.

KRÜGER, K.; SCHOLTZ, C.H. The effect of ivermectin on the development and reproduction of the dungbreeding fly Musca nevilli Kleynhans (Diptera: Muscidae). Agriculture, Ecosystems \& Environment, v.53, p.13-18, 1995.

LEORA SOFWARE. POLO-PC. A user's guide to probit analysis or logit analysis. Berkeley: Leora Software, 1987.

LIMA, L.G.F; PERRI, S.H.V; PRADO, A.P. Efeito do tratamento em dose única ou múltipla com ivermectina na emergencia de Haematobia irritans (L.) (Diptera: Muscidae). Veterinária e Zootecnia, v.16, n.2, p.410-418, 2009.

METCALF, R.L. Changing role of insecticides in crop protection. Annual Review of Entomology, v.25, p.219-256, 1980.

OLIVEIRA, P.C.S.; TREVIJANO, L.K.G.; BELO, M. Sensibilidade estacional em linhagens de $\mathrm{Musca}$ domestica (L.) para três tipos de inseticidas. Anais da Sociedade Entomologica do Brasil, v.22, n.3, p.455-461, 1993.

PEDROSO-DE-PAIVA, D; GOYA, G.E.M. Ivermectin no controle de Sarcopromusca pruna (Shannon \& Del Ponte, 
1926) (Diptera: Muscidae), veiculador dos ovos de Dermatobia hominis L.Jr., 1781 (Diptera: Cuterebridae). Revista Brasileira de Parasitologia Veterinaria, v.3, p.61-64, 1994.

PUTTER, I.; MACCONNELL, J.G.; PRIESTER, F.A.; HAIDRI, A.A.; RISTICH, S.S.; DYBAS, R.A.

Avermectins: novel insecticides, acaricides and nematicides from a soil microorganism. Experientia, v.37, p.963-964, 1981.

ROUSH,R.T.; WRIGHT, J.E. Abamectin: toxicity to house flies resistant to synthetic organic insecticides. Journal of Economic Entomology, v.79, p.562-564, 1986.

RUGG, D.; KOTZE, A.C.; THOMPSON, D.R.; ROSE, H.A. Susceptibility of laboratory-selected and field strains of the Lucilia cuprina (Diptera: Calliphoridae) to ivermectin. Journal of Economic Entomology, v.91, n.3, p.601-607, 1998.

SAS INSTITUTE. SAS User's Guide: Statistics. Cary, North Carolina, USA, 1986.
SCOTT, J.G. Cross-resistance to the biological insecticide abamectin in pyrethroid-resistance house flies. Pesticide Biochemistry and Physiology, v.34, p.2731, 1989.

SINDICATO NACIONAL DA INDÚSTRIA DE PRODUTOS PARA SAÚDE ANIMAL. Brasil. Mercado veterinário por classe terapêutica e espécie animal. São Paulo: SINDAN, 2007. Disponível em: <http:/ / www. sindan.org.br>. Acesso em: 22 mar. 2007.

SILVA, J.J.; MENDES, J. Effect of diflubenzuron on immature stages of Haematobia irritans (L.) (Diptera: Muscidae) in Uberlândia, state of Minas Gerais, Brazil. Memorias do Instituto Oswaldo Cruz, v.97, p.679-682, 2002.

STRONG, L.; BROWN, T.A. Avermectins in insect control and biology: a review. Bulletin of Entomological Research, v.77, p357-389, 1987.

Recebido em 24/4/08

Aceito em 30/5/10 